\title{
Palmprint Recognition Using 3-D Information
}

\author{
David Zhang, Fellow, IEEE, Guangming Lu, Wei Li, Student Member, IEEE, \\ Lei Zhang, Member, IEEE, and Nan Luo
}

\begin{abstract}
Palmprint has proved to be one of the most unique and stable biometric characteristics. Almost all the current palmprint recognition techniques capture the $2-D$ image of the palm surface and use it for feature extraction and matching. Although 2-D palmprint recognition can achieve high accuracy, the 2-D palmprint images can be counterfeited easily and much 3-D depth information is lost in the imaging process. This paper explores a 3-D palmprint recognition approach by exploiting the 3-D structural information of the palm surface. The structured light imaging is used to acquire the 3-D palmprint data, from which several types of unique features, including mean curvature image, Gaussian curvature image, and surface type, are extracted. A fast feature matching and score-level fusion strategy are proposed for palmprint matching and classification. With the established 3-D palmprint database, a series of verification and identification experiments is conducted to evaluate the proposed method. The results demonstrate that 3-D palmprint technique has high recognition performance. Although its recognition rate is a little lower than 2-D palmprint recognition, 3-D palmprint recognition has higher anticounterfeiting capability and is more robust to illumination variations and serious scrabbling in the palm surface. Meanwhile, by fusing the 2-D and 3-D palmprint information, much higher recognition rate can be achieved.
\end{abstract}

Index Terms-Biometrics, 3-D palmprint recognition, feature extraction, surface curvature.

\section{INTRODUCTION}

B IOMETRIC authentication is playing important roles in applications of public security, access control, forensic, banking, etc. [1]. The commonly used biometric characteristics include fingerprint, face, iris, signature, gait, etc. In the past decade, palmprint recognition has grown rapidly, starting from the inked palmprint-based offline methods [2], [3] and developing to the charge-coupled device (CCD) camera-based online methods [4]-[8]. Almost all the current palmprint-recognition techniques and systems are based on the 2-D palm images (inked images or CCD camera-captured images). Although 2-D palmprint recognition techniques can achieve high accuracy, the 2-D

Manuscript received September 1, 2008; revised December 22, 2008. First published June 16, 2009; Current version published August 19, 2009. This work was supported in part by the Hong Kong Special Administrative Region (HKSAR) Government under General Research Fund (GRF), in part by the Hong Kong Polytechnic University under Central Fund, and in part by the National Natural Science Foundation of China (NSFC)/863/Shenzhen - Hong Kong (SZHK)-Innovation Funds under Contract 60620160097, Contract 60803090, Contract 2006AA01Z193, and Contract SG200810100003A. This paper was recommended by Associate Editor E. Trucco.

D. Zhang, L. Zhang, and N. Luo are with the Biometrics Research Center, Department of Computing, The Hong Kong Polytechnic University, Kowloon, Hong Kong (e-mail: cslzhang@ comp.polyu.edu.hk).

W. Li is with the Institute of Image Processing and Pattern Recognition, Shanghai Jiao Tong University, Shanghai 200240, China.

G. Lu is with the Biocomputing Research Center, Shenzhen Graduate School, Harbin Institute of Technology, Shenzhen 518055, China.

Color versions of one or more of the figures in this paper are available online at http://ieeexplore.iee.org.

Digital Object Identifier 10.1109/TSMCC.2009.2020790 palmprint can be counterfeited easily and much 3-D palm structural information is lost in the 2-D palmprint acquisition process. In addition, strong illumination variations and serious scrabbling in the palm may invalidate 2-D palmprint recognition. Therefore, it is of high interest to explore new palmprint recognition techniques to overcome these difficulties. Intuitively, 3-D palmprint recognition is a good solution.

In the online 2-D palmprint recognition system, a CCD camera is used to acquire the palmprint image. The main features in 2-D palmprint include principal lines and wrinkles [4]. Although 2-D palmprint recognition has proved to be efficient in terms of verification rate, it has some inherent drawbacks. First, the palm is not a pure plane and the 3-D depth information cannot be captured by using a single CCD camera. Second, the illumination variations in the system will affect a lot of 2-D palmprint image and may lead to false recognition. Third, although the area of palm is large, too much contamination or too much scrabbling in the palm can still make the recognition invalid. Fourth, the 2-D palmprint image can be copied easily and counterfeited so that the antiforgery ability of 2-D palmprint needs improvement.

Recently, 3-D techniques have been used in biometric authentication, such as 3-D face [9]-[11] and 3-D ear recognition [12], [13]. Range data are usually used in these 3-D biometric applications. Most of the existing commercial 3-D scanners use laser triangulation to acquire the 3-D depth information, for example, the widely used Minolta VIVID Series [10], [12], [13]. Nonetheless, the laser-triangulation-based 3-D imaging technique has some shortcomings with respect to the biometric application. For instance, the resolution of 3-D cloud point may not be high enough for the requirement of accuracy in biometric authentication; on the other hand, if we want to improve the data resolution, the laser scanning speed must be decreased and the real-time requirement in biometric authentication is hard to meet. With the earlier considerations, we propose to use structured light imaging [14], [15], [20], [21] to establish the 3-D palmprint acquisition system. The structured light imaging is able to accurately measure the 3-D surface of an object while using less time than laser scanning. Fig. 1 shows the 3-D palmprint acquisition system developed by the Biometrics Research Center, The Hong Kong Polytechnic University. There is a peg in the developed device serving as a control point to fix the hand. When the user puts his/her palm on the system, an LED projector will generate structured light stripes and project them to the palm. A series of gray-level images of the palm with the stripes on it is captured by the CCD camera, and then the depth information of the palm surface is reconstructed from the stripe images.

Compared with other 3-D biometric characteristics, 3-D palmprint has some desirable properties. For instance, compared with 3-D ear recognition, the palmprint is much more 



Fig. 1. Developed 3-D palmprint authentication system with structured light imaging and an example of the use of the system.

convenient to collect and user-friendly; compared with 3-D face, the users do not need to close or block their eyes, and projecting stripes (or emitting laser light) on palm has much higher acceptability than that on face. In the data acquisition process, the palm can be placed easily so that the collected data are very stable. One disadvantage of 3-D palmprint may be that the palm surface is relatively plane so that the depth information of palm is more difficult to capture than that of face or ear. However, as can be seen in this paper, the curvature features of palm can be well captured by using the developed structured light imaging system. With the proposed feature extraction and matching procedures, the whole 3-D palmprint recognition system can reach very high performance in accuracy, speed, and anticounterfeit capability.

The iterative closest point (ICP) algorithm [16] is widely used in many 3-D object recognition systems for matching. The ICP schemes, however, are not suitable for 3-D palmprint matching because the noise and variations of the palm may have much impact on the matching score. In this paper, we will extract the local curvature features of 3-D palmprint for classification and matching. After the 3-D depth information of palm is obtained, a subarea, called the region of interest (ROI), of the 3-D palmprint image is extracted. Besides reducing the data size for processing, the ROI extraction process also serves to align the palmprints and normalize the area for feature extraction. The mean curvature and Gaussian curvature features of each cloud point in the ROI are then calculated. To save storage space and speed up the matching process, we convert the curvature features to gray-level images, i.e., the mean curvature image (MCI) and Gaussian curvature image (GCI). The third kind of 3-D feature, the surface types (STs), of the palmprint is also defined and extracted. Finally, by fusing the MCI/GCI and ST features, the input palm can be classified and recognized. We established a 3-D palmprint database with 6000 samples from 260 people. Extensive experiments are conducted to evaluate the performance of the proposed feature extraction and matching schemes.

The rest of the paper is organized as follows. Section II describes the acquisition of 3-D palmprint data. Section III discusses the ROI region determination and the 3-D feature ex-

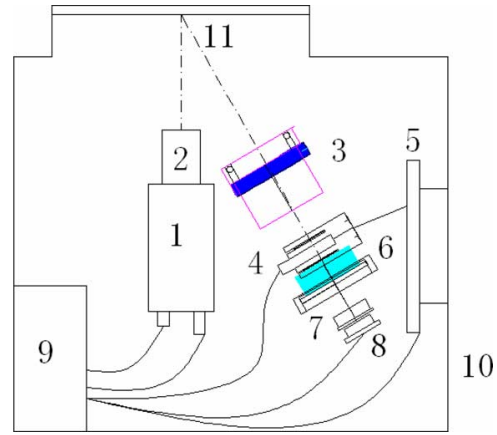

Fig. 2. Architecture of the developed 3-D palmprint data acquisition device: 1) CCD camera; 2) camera lens; 3) casting lens; 4) LCD panel; 5) LCD controller; 6) back convergent lens; 7) front convergent lens; 8) white LED light source; 9) signal and power control box; 10) box shell.

traction from ROI. The calculation of palm curvatures and the generation of GCI, MCI, and ST features are described in detail. Section IV presents the feature matching and fusion methods. Section V shows the experimental results and Section VI concludes the paper.

\section{3-D PALMPRINT DATA ACQUISITION}

The commonly used 3-D imaging techniques include multiviewpoint reconstruction [17], [18], laser scanning [19], and structured light scanning [14], [15], [20]-[24]. Roughly speaking, multiviewpoint reconstruction is a low-cost but lowaccuracy method. It is suitable to measure objects that have obvious corner features. Another problem of multiviewpoint-based 3-D reconstruction is that it may be hard and time-consuming to find the correspondence points in different viewpoint images. Laser scanning is a popular 3-D scanning method and is able to reach high resolution but at the cost of expensive instrument and long collecting time. Particularly, for sweating palms, laser scanning will be greatly affected by the palm surface reflection. Structured light imaging is a well-established 3-D scanning technique. By using phase-shifting method, it can measure the object surface with high accuracy and in a relatively short time. 


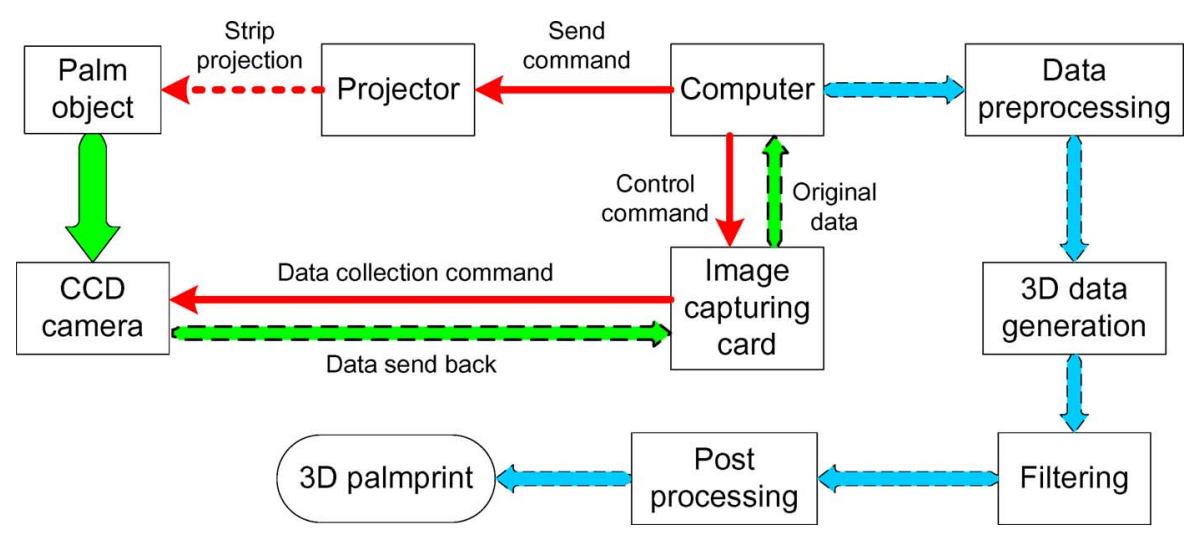

Fig. 3. 3-D palmprint data collection and processing process (red solid line arrows denote sending command, green arrows denote the data transport of collecting, and the blue arrows denote data processing).

Considering the requirements of accuracy and speed in biometric authentication, in the application of 3-D palmprint recognition, we choose to use structured light scanning to acquire the palm depth information. The use of structured light in object surface measurement can be traced back to more than two decades ago [14]. Since then, it has been widely used in many applications, such as 3-D object measurement, 3-D shape reconstruction, reverse engineering, etc., [20]-[22]. In structured light imaging, a light source projects some structured light patterns (stripes) onto the surface of the object. The reflected light is captured by a CCD camera and then a series of images is collected. After some calculation, the 3-D surface depth information of the object can be obtained. In earlier times, parallel light such as laser [23] or point light array [24] were used. With the development of the light source techniques, liquid crystal light projectors have been successfully used as the light source [25]. In our developed system, a cost-effective gray liquid crystal display (LCD) projector with LED light source is employed, and some shift light patterns are projected to the palm.

Fig. 2 shows the architecture of the developed 3-D palmprint data acquisition device. It is mainly composed of a light projecting unit and a data collection unit. The light projecting unit mainly contains a white LED light source, an LCD panel, and several convergent lenses. The data collection unit contains a CCD camera and a camera lens. Fig. 3 illustrates the 3-D palmprint data collection and processing process. The computer controls a projector to project a series of 13 structured light stripes to the palm inner surface and the CCD camera captures the palm images with projected stripes on it. At the same time, the computer sends a command to the data collection board to store the images. The data collection costs about $2 \mathrm{~s}$. From these palm images, the depth information of each point on the palm can be computed using phase transition and phase expansion techniques [15]. These processing, which are marked using blue arrows in Fig. 3, will cost about 0.5 s. So the total time for 3 -D palmprint generation is about $2.5 \mathrm{~s}$.

Fig. 4 illustrates the imaging principle of the structured light technique [14]. Interested readers can refer to [14] for more details about structured light imaging. In Fig. 4, there is a reference plane whose height is 0 . By projecting light through grating to the object surface, the relative height of a point $D$ at

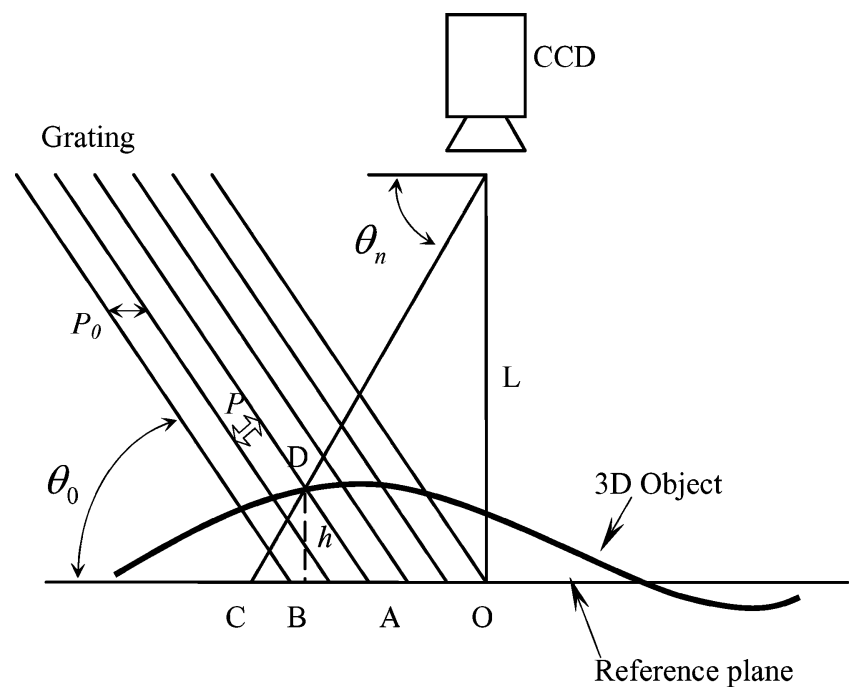

Fig. 4. Principle of structured light imaging.

spatial position $(x, y)$ to the reference plane can be calculated as follows [14]:

$$
h(x, y)=\overline{B D}=\frac{\overline{A C} \tan \theta_{0}}{1+\tan \theta_{0} / \tan \theta_{n}}
$$

with

$$
\overline{A C}=\frac{\phi_{C D}}{2 \pi} P_{0}
$$

where $P_{0}$ is the wavelength of the projected light on the reference plane, $\theta_{0}$ is the projecting angle, $\theta_{n}$ is the angle between the reference plane and the line that passes through the current point and the CCD center, and $\phi_{C D}$ is the phase difference between points $C$ and $D$. Because the phase of point $D$ on the 3-D object is equal to the phase of point $A$ on the reference plane, $\phi_{C D}$ can be calculated as

$$
\phi_{C D}=\phi_{C A}=\phi_{O C}-\phi_{O A} .
$$

By using (1) and the phase shifting and unwrapping technique [15], we can retrieve the depth information of the object surface by projecting a series of phase stripes on it (13 stripes are used 

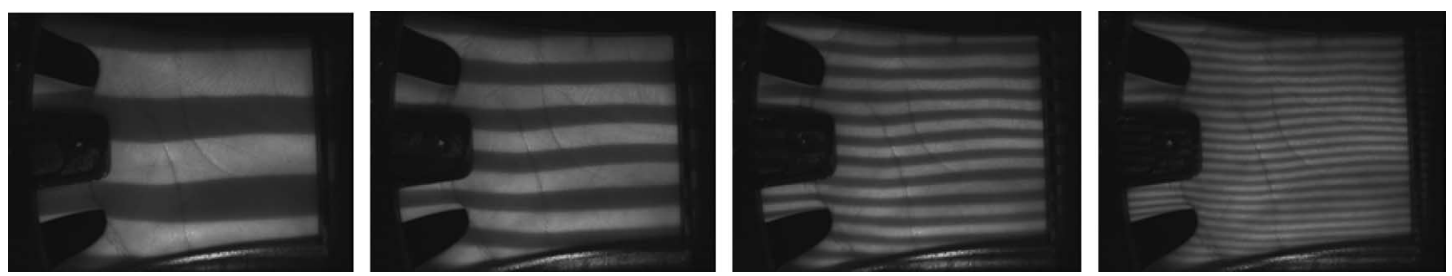

Fig. 5. Sample patterns of the stripes on the palm.

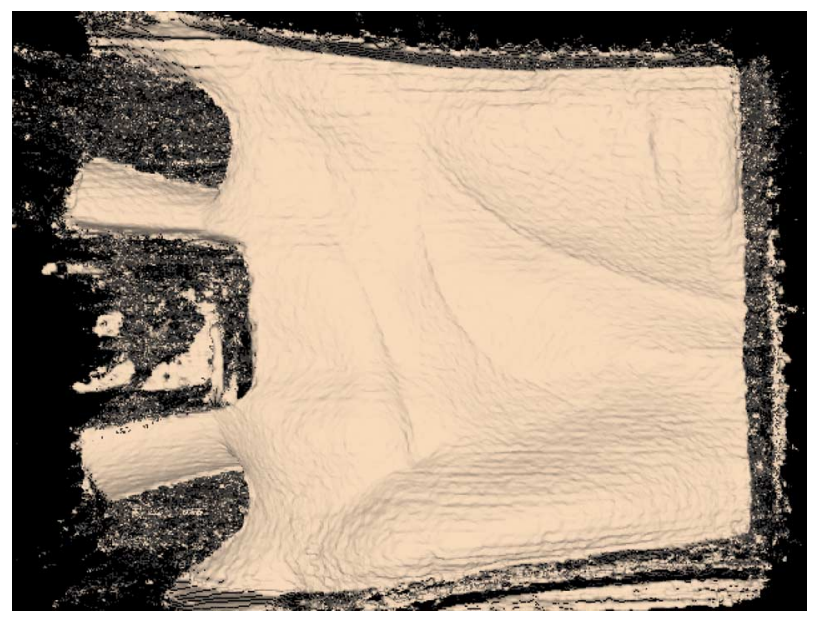

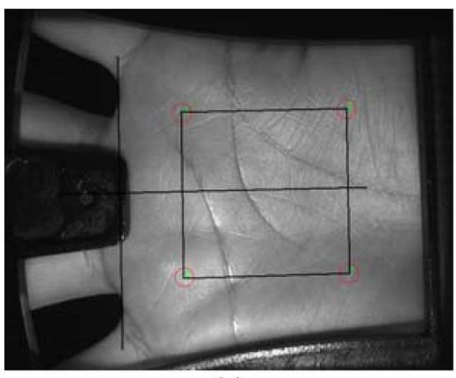

(a)

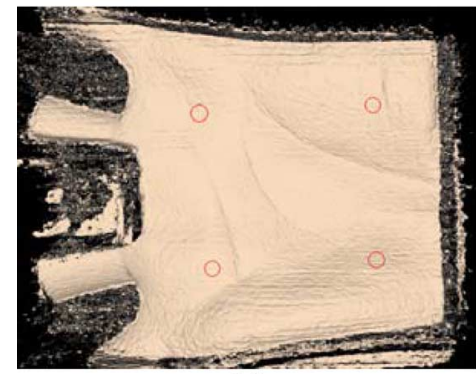

(c)



(b)



(d)

With this processing, the relative height of each point $h(x, y)$ could be calculated. The range data of the palm surface can then be obtained. In the developed system, the size of the 3-D image is $768 \times 576$ with 150 dpi resolution, i.e., there are totally 442368 cloud points to represent the 3-D palmprint information. And the depth precision of the 3-D image is between 0.05 and $0.1 \mathrm{~mm}$. Fig. 6 shows an example 3-D palmprint image captured by the system. The gray level in Fig. 6 is related to the value of $h(x, y)$, and it is rendered by OpenGL automatically for better visualization.

\section{FeAtURE EXTRACTION From 3-D PALMPRINT}

\section{A. ROI Extraction}

From Fig. 6, we can see that in the 3-D palmprint image of resolution $768 \times 576$, many cloud points, such as those in the boundary area and those in the fingers, could not be used in feature extraction and recognition. Most of the useful and stable features are located in the center area of the palm. In addition, at different times when the user puts his/her hand on the system, there will be some relative displacements of the positions of the palm, even if we impose some constraints on the users to place their hands. Therefore, before feature extraction, it is necessary to perform some preprocessing to align the palmprint and extract the central area of it, which is called the ROI.

By using the developed structured-light-based 3-D imaging system, the 2-D and 3-D palmprint images can be obtained simultaneously, and there is a one-to-one correspondence be-

Fig. 7. ROI extraction of 3-D palmprint from its 2-D counterpart. (a) 2-D palmprint image, the adaptively established coordinate system, and the ROI (i.e., the rectangle). (b) Extracted 2-D ROI. (c) 3-D palmprint image, whose cloud points have a one-to-one correspondence to the pixels in the 2-D counterpart. (d) Obtained 3-D ROI by grouping the cloud points corresponding to the pixels in 2-D ROI. (Note that we change the viewpoint for better visualization.)

tween the 3-D cloud points and the 2-D pixels. Therefore, the ROI extraction of the 3-D palmprint data can be easily implemented via the 2-D palmprint ROI extraction procedure. In this paper, we use the algorithm in [4] to extract the 2-D ROI. Once the 2-D ROI is extracted, the 3-D ROI is obtained by grouping the cloud points that are in correspondence to the pixels in the 2-D ROI. Fig. 7 illustrates the ROI extraction process. Fig. 7(a) shows a 2-D palmprint image, the established local coordinate system by using the algorithm in [4], and the ROI (i.e., the rectangle); Fig. 7(b) shows the extracted 2-D ROI; Fig. 7(c) shows the 3-D palmprint image, and Fig. 7(d) shows the obtained 3-D ROI by grouping the cloud points corresponding to the pixels in 2-D ROI. (Note that we change the viewpoint for better visualization.)

By using the ROI extraction procedure, the 3-D palmprints are aligned so that the small translation and rotation introduced in the data acquisition process are corrected. In addition, the data amount used in the following feature extraction and matching process is significantly reduced. This will save much computational cost. Fig. 8(a) shows the extracted 3-D ROIs of 


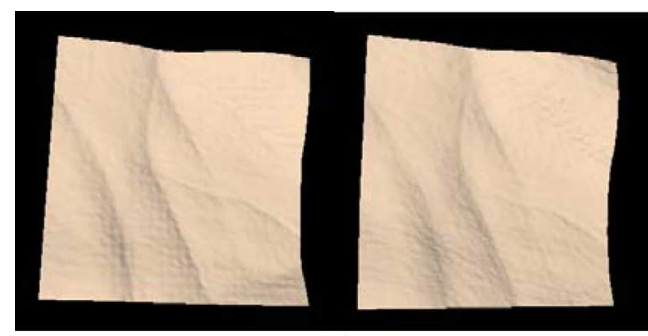

(a)

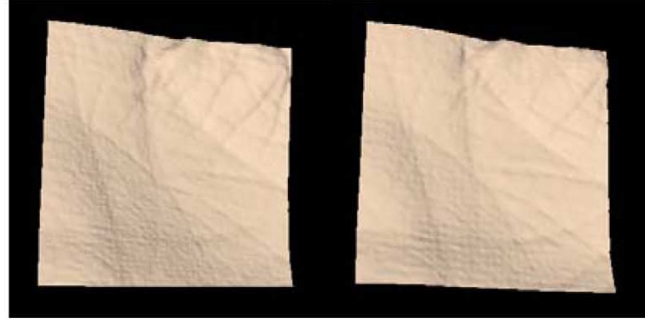

(b)

Fig. 8. (a) 3-D ROIs extracted from two palmprints of one person. (b) 3-D ROIs extracted from two palmprints of another person.
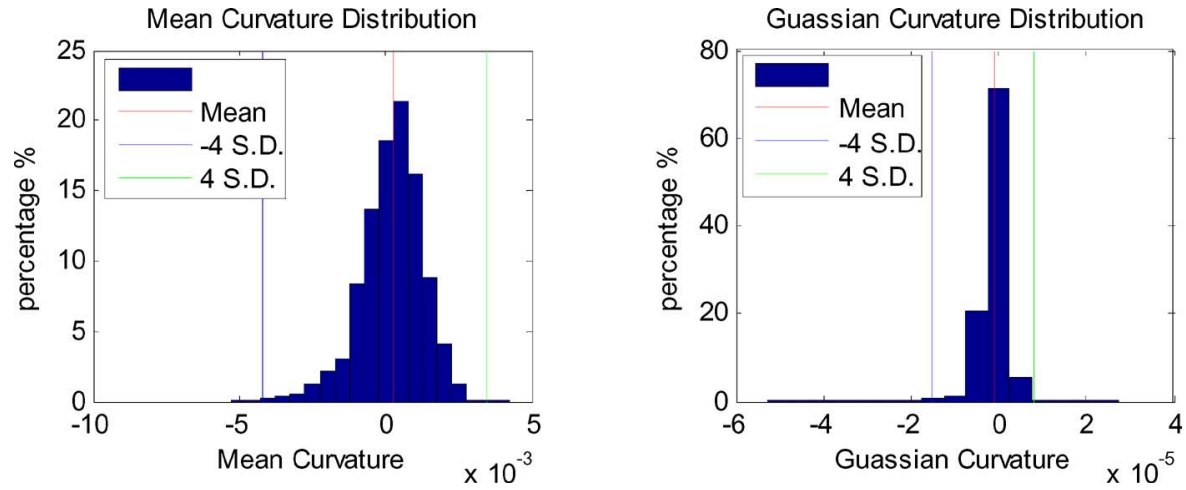

Fig. 9. Distributions of mean and Gaussian curvatures of 3-D palmprint images.

two palmprints from the same person. Fig. 8(b) shows another example.

\section{B. Curvature Calculation}

With the obtained ROI, stable and unique features are expected to be extracted for the following pattern matching and recognition. The depth information in the acquired 3-D palmprint reflects the relative distance between the reference plane and each point in the object (referring to Fig. 4). As can be seen in Fig. 1, most of the palm region, which will be captured by the system for personal recognition, will not touch the device. Therefore, there is little pressure on the palm. In data acquisition, the users are asked to put their hands naturally on the device. Thus, the pressure-caused deformation of the palmprint image is actually very small. The $z$-values of the 3-D cloud points are mainly affected by noise and the pose change of hand in scanning. The ROI extraction process can only correct the rotation and translation displacements in the $x-y$ plane but not the $z$-axis. The noise and variations in the 3-D palmprint cloud points make the well-known ICP algorithms [16] not suitable for 3-D palmprint recognition. Instead, the local invariant features, such as the curvatures [26] of the principal lines and strong wrinkles in the palm surface, will be much more stable in representing the characteristics of 3-D palmprint.

Let $p$ be a point on the surface $S$. Consider all curves $C_{i}$ on $S$ passing through the point $p$. Each curve $C_{i}$ will have an associated curvature $K_{i}$ at $p$. Among those curvatures $K_{i}$, at least one is characterized as maximal $k_{1}$ and one as minimal $k_{2}$, and these two curvatures $k_{1}$ and $k_{2}$ are known as the principal curvatures of point $p$ on the surface [26]. The mean curvature
$H$ and the Gaussian curvature $K$ of $p$ are defined as follows:

$$
H=\frac{1}{2}\left(k_{1}+k_{2}\right) \quad K=k_{1} * k_{2}
$$

The mean and Gaussian curvatures are intrinsic measures of a surface, i.e., they depend only on the surface shape but not on the way how the surface is placed in the 3-D space [26]. Thus, such curvature features are robust to the rotation, translation, and even some deformation of the palm. The captured 3-D palmprint data are organized range data. We adopt the algorithm in [27] for its simplicity and effectiveness in estimating the mean and Gaussian curvatures. For more information, refer to [27].

\section{C. $M C I$ and GCI}

With the method in [27], the mean and Gaussian curvatures of a 3-D palmprint image can be calculated. Fig. 9 shows the distribution of the mean and Gaussian curvatures of $1003-\mathrm{D}$ palmprint images.

For better visualization and more efficient computation, we convert the original curvature images into gray-level integer images according to the distributions. We first transform the curvature image $C$ (Gaussian curvature $K$ or mean curvature $H$ ) into $\bar{C}$ as follows:

$$
\bar{C}(i, j)=0.5 \frac{C(i, j)-\mu}{4 \delta}+0.5
$$

where $\mu$ is the mean of the curvature image. With (5), most of the curvature values will be normalized into the interval $[0,1]$. 


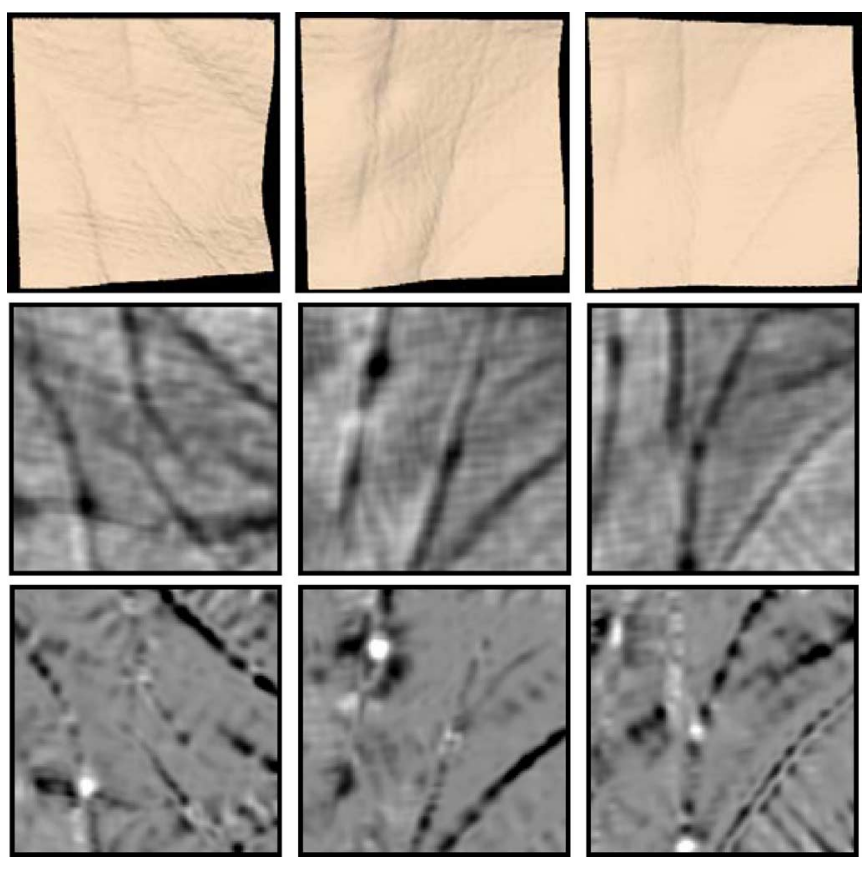

Fig. 10. 3-D ROI images (first row) of three different palmprints and their MCI (second row) and GCI (third row) images. From left to right, each column shows the images of one palm.

We then map $\bar{C}(i, j)$ to an 8-bit gray-level image $G(i, j)$

$$
G(i, j)= \begin{cases}0, & \bar{C}(i, j) \leq 0 \\ \operatorname{round}(255 \times \bar{C}(i, j)), & 0<\bar{C}(i, j)<1 \\ 255, & \bar{C}(i, j) \geq 1\end{cases}
$$

We call images $G(i, j)$ the MCI and GCI, respectively for mean and Gaussian curvatures. Fig. 10 illustrates the MCI and GCI images of three different palms and Fig. 11 depicts the MCI and GCI images of a palm at different acquisition times. We can see that the 2-D MCI and GCI images can well preserve the 3-D palm surface features. Not only the principal lines, which are the most important texture features in palmprint recognition, are clearly enhanced in MCI/GCI, but also the depth information of different shape structures is well preserved. The MCI/GCI feature images provide us a good basis for further processing and pattern matching.

\section{Points Classification Using STS}

Since the palm is a continuous surface with different convex and concave structures, we can classify the points in the palm into different groups based on their local surface characteristics. Such kind of 3-D feature is called ST, and it can be determined by the signs of mean and Gaussian curvature values [28]. In [27], eight fundamental STs were defined. Fig. 12 illustrates the shapes of the eight STs and Table I lists the definition of them based on the corresponding mean curvature $H$ and Gaussian curvature $K$. In total, nine STs can be defined, including the eight fundamental STs in Fig. 12 and another special case for $H=0$ and $K>0$. Using STs, the points in the 3-D palmprints can be intuitively classified into nine classes. For example, the



Fig. 11. 3-D ROI images (first row) of the same palmprint but collected at different times and their MCI (second row) and GCI (third row) images. From left to right, each column shows the images for each time.


Valley


Flat

Fig. 12. Eight fundamental STs defined by their different convex and concave structures [27].

GCI images in Figs. 10 and 11 have some white pixels, which implies $K>0$; meanwhile, the corresponding points in the MCI images are black, which implies $H<0$. From Table I, we know that these points belong to the PEAK ST $(\mathrm{ST}=1)$.

We need to quantize the values of $H$ and $K$ to fix the intervals that make $H=0$ or $K=0$. This can be simply implemented 
TABLE I

ST LABELS From CURVATURE SignS

\begin{tabular}{|l|l|l|l|}
\hline & $K>0$ & $K=0$ & $K<0$ \\
\hline \multirow{2}{*}{$H<0$} & Peak & Ridge & Saddle Ridge \\
& ST=1 & ST=2 & ST=3 \\
\hline \multirow{2}{*}{$H=0$} & None & Flat & Minimal Surface \\
& ST=4 & ST=5 & ST=6 \\
\hline \multirow{2}{*}{$H>0$} & Pit & Valley & Saddle Valley \\
& ST=7 & ST=8 & ST=9 \\
\hline
\end{tabular}

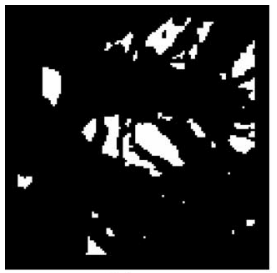

(a)

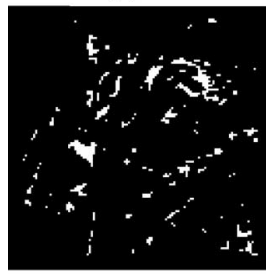

(d)

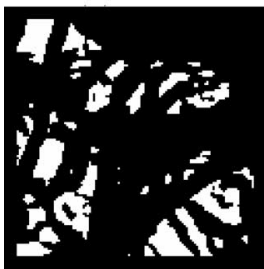

(g)

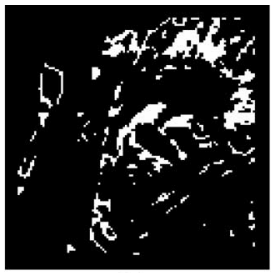

(b)

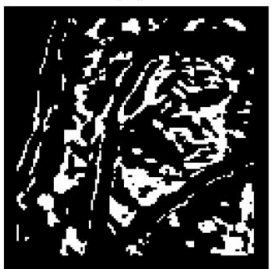

(e)

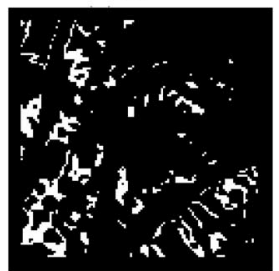

(h)

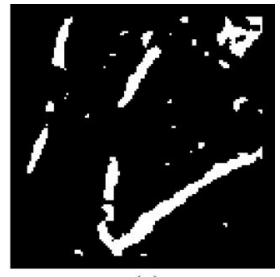

(c)



(f)



(i)
Fig. 13. STs $1-9$ of a palm using binary representation. (a) $\mathrm{ST}=1$. (b) $\mathrm{ST}=$ 2. (c) $\mathrm{ST}=3$. (d) $\mathrm{ST}=4$. (e) $\mathrm{ST}=5$. (f) $\mathrm{ST}=6$. (g) $\mathrm{ST}=7$. (h) $\mathrm{ST}=8$. (i) $\mathrm{ST}=9$.

by using two thresholding parameters $\varepsilon_{H}$ and $\varepsilon_{K}$

$$
\begin{cases}H(i, j)=0, & \text { if }|H(i, j)|<\varepsilon_{H} \\ K(i, j)=0, & \text { if }|K(i, j)|<\varepsilon_{K}\end{cases}
$$

The thresholds $\varepsilon_{H}$ and $\varepsilon_{K}$ should be adaptive to different palms. To this end, we normalize the mean or Gaussian curvature $C(i, j)$ by its standard deviation as

$$
C_{s}(i, j)=\frac{C(i, j)}{2 \delta} .
$$

Using (8), most curvature values will fall into the interval $[-1,1]$ without changing their signs. Then, we can easily set the thresholds $\varepsilon_{H}$ and $\varepsilon_{K}$ around zero in (7).

With the aforementioned procedures, each point in the 3-D palmprint can be classified into one of the nine STs. Fig. 13 shows the classification results of a palm sample using binary images, i.e., a white pixel in an ST image means that it belongs to the corresponding ST. These features can be used separately or jointly in the matching process.
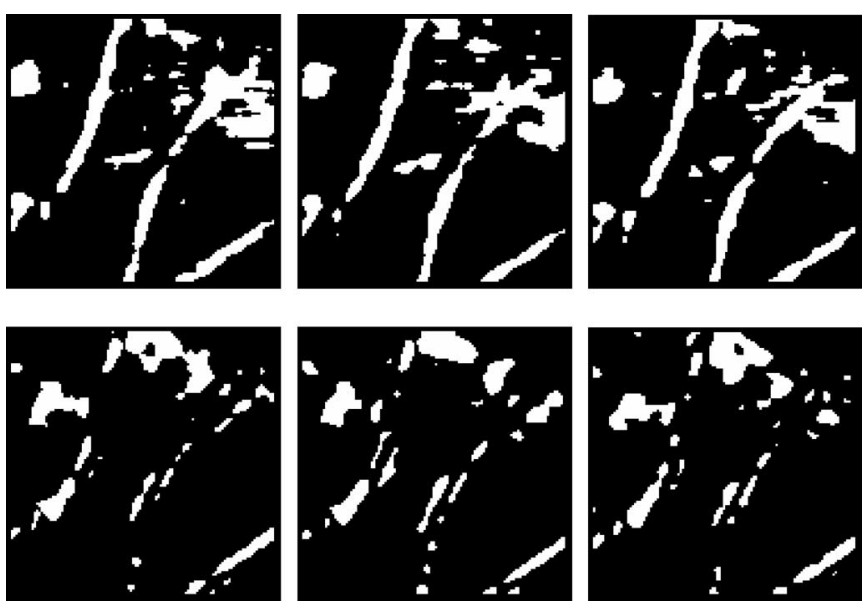

Fig. 14. Binarized (first row) MCI images and (second row) GCI images.

\section{FeAture Matching AND Fusion}

Using the techniques developed in Section III, the MCI, GCI, and ST features of the 3-D palmprint can be extracted. The different palmprints can then be matched based on those features and the recognition can be accomplished according to the matching score. In this section, we discuss the matching strategy of different features and their fusion. We will then discuss the fusion of 2-D and 3-D palmprint information for higher recognition rates.

\section{A. MCI/GCI Feature Matching}

The extracted MCI/GCI features (referring to (6)) are 8-bit gray-level images. The variations of the local curvatures are mainly caused by acquisition noise and the pose changes of the hand. To reduce such variations and extract the stable and intrinsic curvature features, we binarize the MCI and GCI maps in the following processing. The principal lines and strong wrinkles are the most stable and significant features in the palmprint. After the binarization, they could be well preserved in the MCI and GCI maps, while the noise-caused small variations are removed. In addition, the binarization of the MCI and GCI maps can make feature matching very fast. Here, we simply convert the MCI/GCI into binary images by using adaptive thresholding

$$
B(i, j)= \begin{cases}1, & G(i, j)<c \times \mu_{G} \\ 0, & \text { others }\end{cases}
$$

where $c$ is a constant and $\mu_{G}$ is the mean value of $G(i, j)$. With our experimental experience, we set $c=0.7$ in the experiments. Fig. 14 shows the binarized versions of the MCI/GCI images in Fig. 11.

We use the AND operation to calculate the matching score of MCI/GCI features. Let $B_{d}$ denote the binarized MCI/GCI image in the database, and by $B_{t}$, the input MCI/GCI binary image. Suppose the image size is $n \times m$. The matching score between $B_{d}$ and $B_{t}$ is defined as

$$
R_{C}=\frac{2 \sum_{i=1}^{n} \sum_{j=1}^{m} B_{d}(i, j) \oplus B_{t}(i, j)}{\sum_{i=1}^{n} \sum_{j=1}^{m} B_{d}(i, j)+\sum_{i=1}^{n} \sum_{j=1}^{m} B_{t}(i, j)}
$$






Fig. 15. Illustration of the 2-D and 3-D feature-level fusion. (a) 2D ROI. (b) 3D ROI. (c) MCI. (d) Energy image of MCI. (e) Enhanced 2D ROI.

where symbol " $\oplus$ " means the AND logic operation. If the two $\mathrm{MCI} / \mathrm{GCI}$ binary feature images $B_{d}$ and $B_{t}$ are the same, then we have $R_{c}=1$; the minimum value of $R_{c}$ is 0 , which means that the two binary images have no overlap "1" pixel.

Since there may still be some displacements between the two palmprint images even after ROI extraction, when calculating the matching score by (10), we will shift two, four, six, and eight pixels of the test image along eight directions: right, left, up, down, left-up, left-down, right-up, and right-down, respectively. Thus, we will have $8 \times 4+1=33$ matching scores and the maximum one is selected.

\section{B. ST Feature Matching}

For each 3-D palmprint, we have nine binary ST images, representing different $\mathrm{STs}$ of the points in it. Let $\mathrm{ST}_{k}^{d}, k=$ $1,2, \ldots, 9$, be the ST images in the database and denote by $\mathrm{ST}_{k}^{t}$ the test ST images. Different from the matching score of $\mathrm{MCI} / \mathrm{GCI}$ features, here we use the absolute value of difference to measure the distance between the two palmprints

$$
R_{\mathrm{ST}}=1-\frac{\sum_{k=1}^{9} \sum_{i=1}^{n} \sum_{j=1}^{m}\left|\mathrm{ST}_{k}^{d}(i, j)-\mathrm{ST}_{k}^{t}(i, j)\right|}{2 \times m \times n} .
$$

If the ST features of two palmprints are identical, we have the maximum matching score $R_{\mathrm{ST}}=1$; on the contrary, if the ST features are extremely different, the ST matching score will be $R_{\mathrm{ST}}=0$.

\section{Matching Score Fusion of MCI, GCI, and ST}

Using (10) and (11), three matching scores (two $R_{c}$ scores for MCI and GCI, respectively, and one $R_{\mathrm{ST}}$ score for $\mathrm{ST}$ ) can be calculated fast. Each one of them can be used to make a decision and the three decisions can be fused so as to reach a final decision. Another way is to fuse the three matching scores first, and then the decision is made based on the fused matching score. Here, we adopt the second strategy.

Suppose there are $n$ matching scores and we denote them by $R_{i}, i=1,2, \ldots, n$. The commonly used scorelevel fusion techniques include Min-Score(MIN) $R_{\mathrm{MIN}}=$ $\min \left(R_{1}, R_{2}, \ldots, R_{n}\right)$, Max-Score(MAX) $R_{\mathrm{MAX}}=\max \left(R_{1}\right.$, $\left.R_{2}, \ldots, R_{n}\right)$, Summation (SUM) $R_{\mathrm{SUM}}=(1 / n) \sum_{i=1}^{n} R_{i}$, and weighted average (WA) methods [29], [30]. We can see that the mean curvature contains more useful information than
Gaussian curvature so that the MCI feature can lead to better matching result than GCI (referring to Section V-C). Therefore, we should assign a greater weight to MCI than to GCI. Because the equal error rate (EER) is an important index of the matching result and it can be estimated by test database, the weights can be determined according to the corresponding EER values.

In [29], a WA scheme, called matcher weighting (MW), is proposed

$$
R_{\mathrm{MW}}=\sum_{i=1}^{n} w_{i} R_{i}, \quad w_{i}=\frac{1 / e_{i}}{\sum_{j=1}^{n} 1 / e_{i}}, \quad i=1,2, \ldots, n
$$

where $w_{i}$ is the weight of $R_{i}$, and $e_{i}$ is the corresponding EER. The MW scheme assigns smaller weights to those features with higher EER values. In [29], a user weighting (UW) fusion scheme was also proposed. The idea comes from the wolf-lamb concept introduced by Doddington [31]. The UW method assigns different weights to different users of different matchers according to the user's inimitable property. The user who can be imitated easily is called as lamb and will be assigned with lower weight. However, in practice, whether the user is a lamb or not highly depends on the database, which limits the application of UW. In our experiments in Section V, we will test the MIN, MAX, SUM, and MW fusion methods.

\section{Fusion of 2-D and 3-D Palmprint Information}

Intuitively, the 2-D and 3-D palmprint information can be fused for better recognition accuracy. The fusion can be performed on either matching score level or feature level. For score-level fusion, we regard each pair of 2-D and 3-D matching scores as a 2-D vector, and then use the linear SVM method [33] to classify the genuine and impostor. Besides score-level fusion, feature-level fusion is also applicable to 2-D and 3-D palmprint to improve the matching performance. The main features in 2-D palmprint are principal lines and wrinkles [4], and these features can also be represented by 3-D palmprint. Sometimes the key features in 2-D image may not be well captured (e.g., overillumination), but these critical discriminant features can be enhanced by fusing with the 3-D information. Here, we use an example shown in Fig. 15 to illustrate the proposed feature-level fusion scheme. Fig. 15(a) is a 2-D ROI that is overilluminated; (b) is the corresponding 3-D ROI; (c) is the MCI feature extracted from the 3-D ROI; (d) is the energy image of the MCI; and (e) 

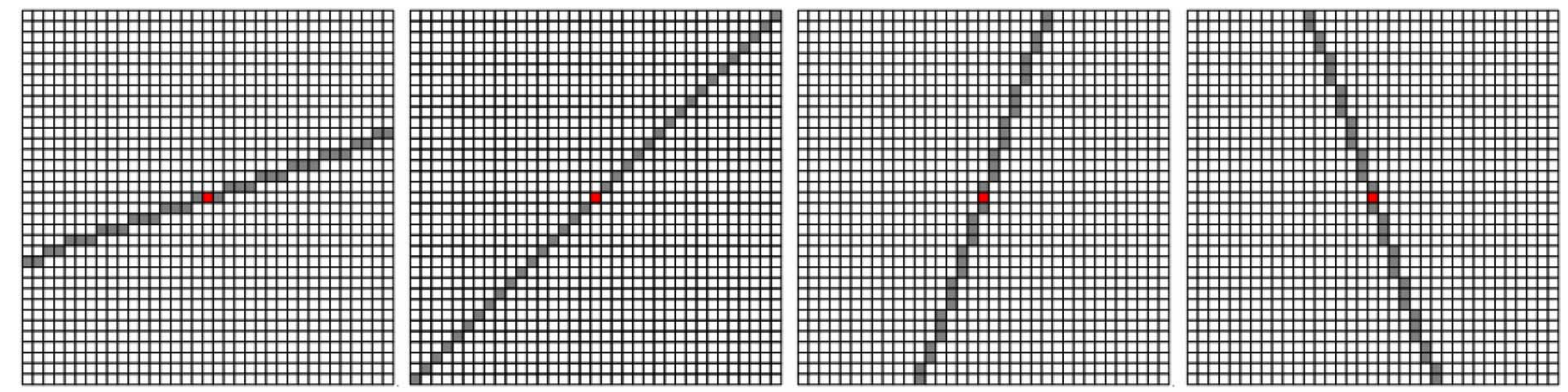

Fig. 16. Example line structure templates used in this paper.

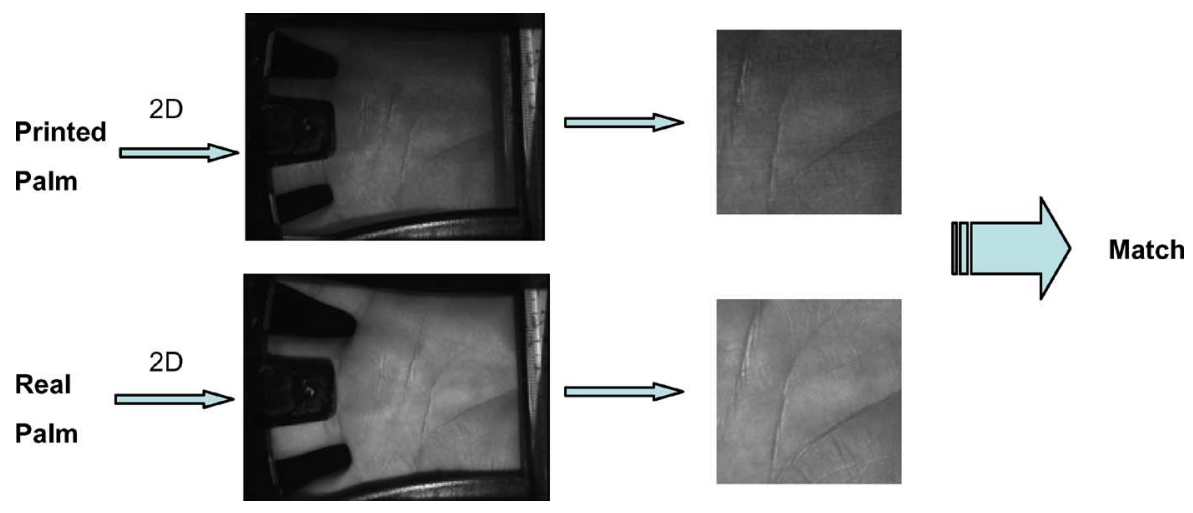

Fig. 17. Anticounterfeiting test of printed palm using 2-D palmprint recognition.



Fig. 18. Anticounterfeiting test of printed palm using 3-D palmprint recognition.
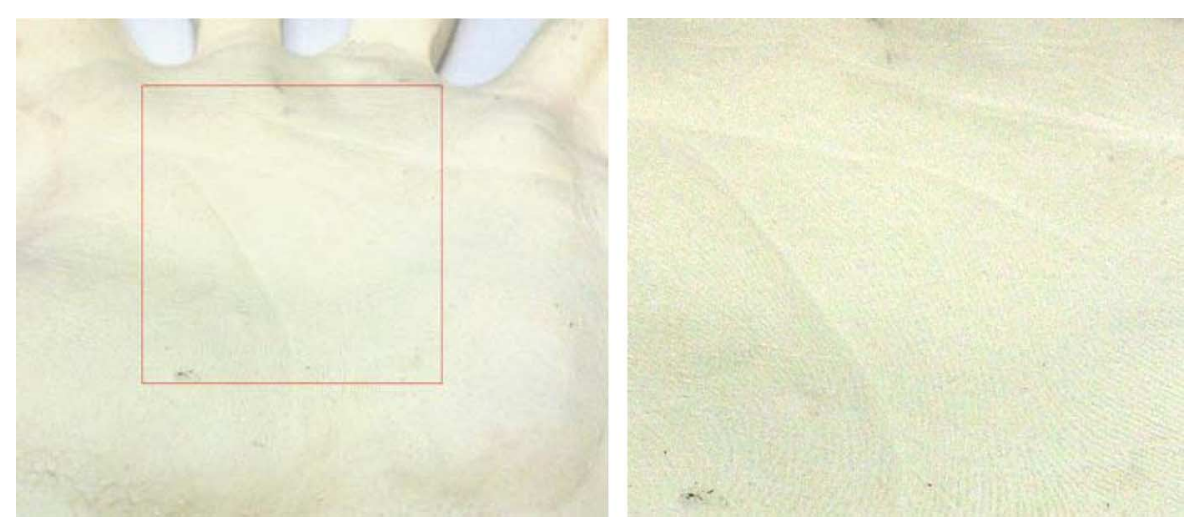

Fig. 19. 3-D plaster palmprint. 


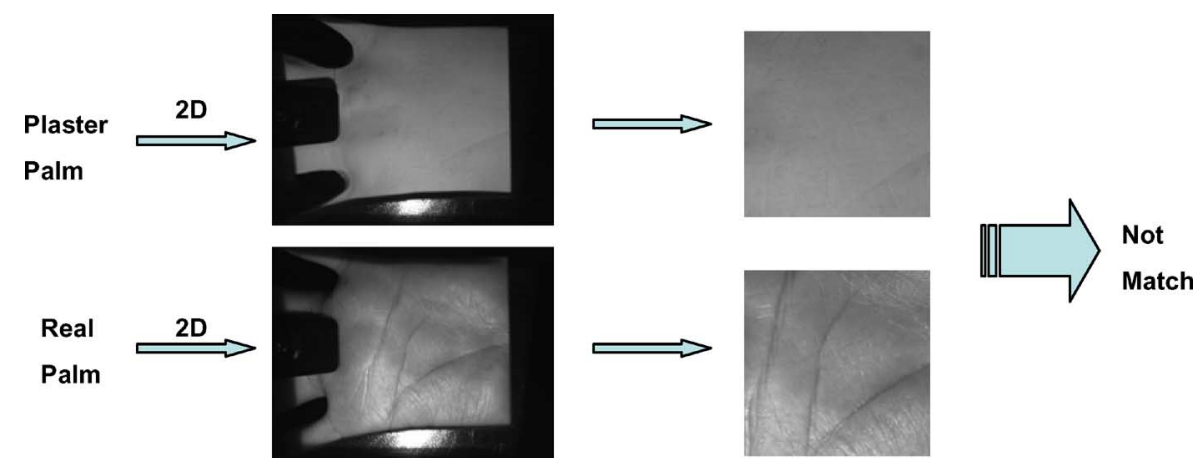

Fig. 20. Anticounterfeiting test of plaster palm using 2-D palmprint recognition.

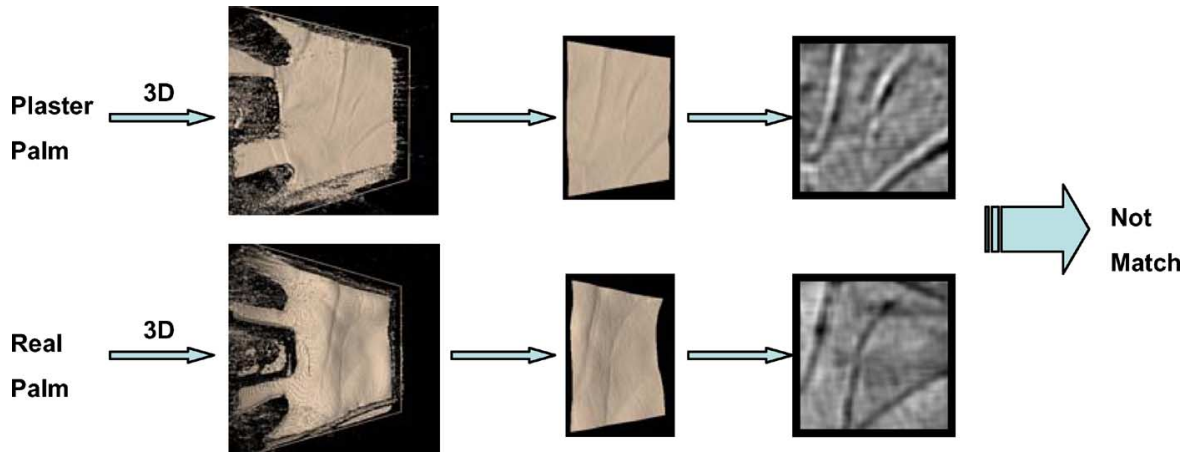

Fig. 21 Anticounterfeiting test of plaster palm using 3-D palmprint recognition.
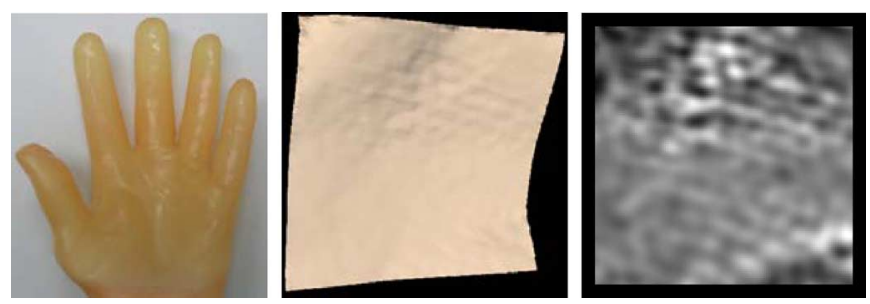

Fig. 22. Counterfeiting palm made by olefin. From left to right: the counterfeiting palm, 3-D ROI, and the MCI of the counterfeiting palm.

is the enhanced 2-D ROI by the 3-D MCI energy image. Here, we use the method in [32] to calculate the energy image of the MCI map.

Let $H$ denote the $m \times n$ MCI map. Then, the energy image of $H$, denoted by $E$, is calculated as

$$
\begin{aligned}
E(i, j) & =\min \left\{e_{i, j}^{1}, \ldots, e_{i, j}^{k}, \ldots, e_{i, j}^{K}\right\}, \\
i & =1, \ldots, m, j=1, \ldots, n
\end{aligned}
$$

with

$$
e_{i, j}^{k}=\sum_{(u, v) \in L_{i, j}^{k}} H(u, v)
$$

where $L_{i, j}^{k}$ is a predefined template centered at $(i, j)$ and $K$ is the total number of templates. Some templates used in this paper are given in Fig. 16. They are basically the templates to model the line structures of different orientations. After calculating the energy image $E$, we enhance the 2-D ROI, denoted by $F$, as follows:

$$
F_{E}(i, j)=F(i, j)-\alpha \times E(i, j)
$$

where $\alpha$ is a parameter to control the fusion and we set $\alpha=$ 0.3 in our experiments. In Fig. 15(e), we can see that the line features are much improved after fusing with the MCI map. They will be well extracted in the following 2-D palmprint feature extraction process and will lead to more robust matching results. Our experimental results in Section V-C validate this.

\section{EXPERIMENTAL RESULTS}

\section{A. Anticounterfeiting Test}

The proposed 3-D palmprint recognition system uses the structured light technique to capture the palm surface depth information. One clear advantage of 3-D palmprint recognition over 2-D palmprint is that it has higher anticounterfeiting capability because a 3-D palm is much harder to forge. In this section, we made anticounterfeiting tests by using printed 2-D palms and 3-D palms made of plaster and olefin. A user is registered in both the 2-D palmprint and 3-D palmprint systems.

We first printed out a 2-D palmprint image of this user on a piece of paper using a high-quality laser printer (HP LaserJet 1020plus, $1200 \mathrm{dpi}$ ). Then, we put this paper on the two systems as input palm to see if it can pass the systems. Figs. 17 and 18 illustrate the test and the recognition results. For 2-D system, we used the competitive coding method [5]. In Fig. 17, we can see that because the printed palm is a pure paper plane and the real palm is a curved surface, the captured palmprint images 

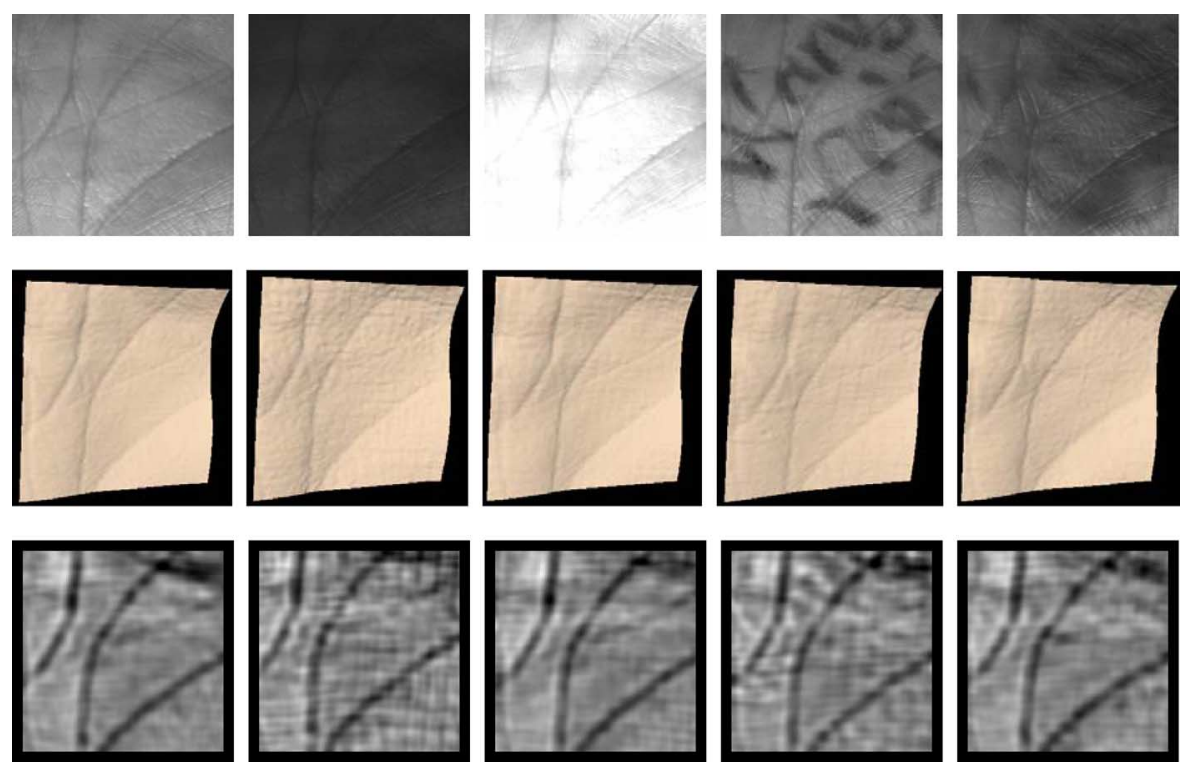

Fig. 23. Sample images in the robustness test database. From top to bottom: 2-D ROI, 3-D ROI, and the 3-D MCI map. From left to right are the five test cases: normal, severe underillumination, severe overillumination, scrabbling, and dirty.

TABLE II

Collecting Conditions of THE Five Test CASES

\begin{tabular}{|l|l|l|l|}
\hline & Aperture value & Scrabbling & Clean/dirty \\
\hline Normal & f 5.6 & No & Clean \\
\hline Severely under illumination & f 11 & No & Clean \\
\hline Severely over illumination & f 2.8 & No & Clean \\
\hline Scrabbling & f 5.6 & Yes & Clean \\
\hline Dirty & f 5.6 & No & Dirty \\
\hline
\end{tabular}

TABLE III

EER OF 2-D AND 3-D PALMPRINT VERIFICATION ON THE ROBUSTNESS TEST DATABASE

\begin{tabular}{|l|l|l|}
\hline & $2 \mathrm{D}$ & $3 \mathrm{D}$ \\
\hline EER & $5.478 \%$ & $0.344 \%$ \\
\hline
\end{tabular}

from printed palm and real palm will have some differences in the illumination level. However, the features (principal lines, wrinkles, etc.) of the two images are identical and the system will successfully match the printed palm with the palmprints in the database because the 2-D palmprint recognition algorithms can deal with the illumination variations to some extent. As expected, it can be seen in Fig. 18 that the 3-D palmprint technique can easily tell the counterfeited palm because there is no depth variation information in the printed paper and the captured 3-D palmprint image from the printed 2-D palmprint is just a noise image.

We made two kinds of 3-D counterfeiting palms by plaster and olefin, respectively, to test whether they can break the 3-D palmprint system. For the plaster counterfeiting palm, we first press a real palm on plasticine to record the palm depth information, and then mold the liquid plaster into the plasticine model to make the 3-D palmprint counterfeit, which is shown in Fig. 19. The anticounterfeit test on this plaster palm is illustrated in Figs. 20 and 21. We can see that both the 2-D and 3-D systems reject the plaster palm. The 2-D system rejects the fake palm because the line features captured in the 2-D image are weak, as shown in Fig. 20. The 3-D system also rejects the fake palm. This is because there is some deformation on the palm surface in the making of the plaster palm. Therefore, the extracted curvature features of the plaster palmprint are very different from those of the real palmprint, as illustrated in Fig. 21. Similar results have been obtained for the counterfeiting test on the olefin palm because it is hard to counterfeit the detailed 3-D information of the real palm. Fig. 22 shows the olefin counterfeiting palm and its 3-D ROI and MCI map. From these tests, we can see that the 3-D system is hard to be spoofed. Certainly, people can make a more "real" 3-D palm to break the system, but the cost will be much higher.

\section{B. Robustness Test: Illumination, Scrabbling, and Dirty}

It is necessary to test the robustness of the proposed 3-D palmprint technique to the variations of illumination, scrabbling, and dirty in the palm. For example, the palm of labor workers may be seriously contaminated when working. Sometimes, one may write down some information on the palm when no paper is at hand. It has been reported that in 2-D palmprint recognition, light- to medium-level illumination variation and palm scrabbling will not affect the recognition accuracy too much [4]. However, when the illumination varies too much or the palm is seriously scrabbled, we can imagine that the 2-D palmprint will not work anymore because the 2-D palmprint features (principal lines, wrinkles, etc.) are hard to capture.

Due to the different imaging principles, the 3-D palmprint technique has clear advantages over its 2-D counterparts in reducing the interference of illumination, scrabbling, and dirty. To prove this declaration, we build a robustness test database that contains 400 samples of both 3-D and 2-D palmprints collected from 40 palms of 20 individuals. For each palm, ten samples were collected: two normal samples, two severely underilluminated samples, two severely overilluminated samples, 




(a)

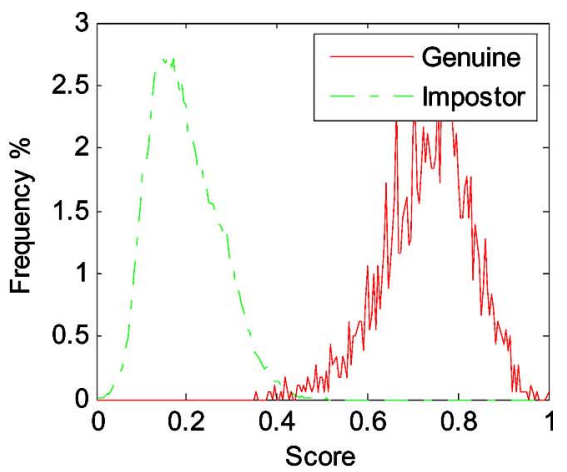

(b)

Fig. 24. Genuine and imposter distributions on the robustness test database. (a) 2-D score distribution. (b) 3-D score distribution.

two scrabbling samples, and two dirty samples. We simulate the illumination variations by changing the aperture value of the camera lens. Fig. 23 shows examples of the 2-D ROI, 3-D ROI, and 3-D MCI of one palm under the five different cases and Table II lists the collecting conditions of the five cases.

Then, verification experiments were performed on this database. Each 3-D/2-D sample was matched with all the other 3-D/2-D samples in the database. A successful matching is called intraclass matching or genuine if the two samples are from the same class. Otherwise, the unsuccessful matching is called interclass matching or impostor. Using the established database, there are 79,800 matchings in total. The 3-D matching score is obtained by fusing the MCI, GCI, and ST at score level (referring to Section IV-C). The 2-D matching score is calculated by using the competitive coding method [5]. Table III lists the EER values of 2-D and 3-D palmprint verification. Fig. 24 shows the distributions of 2-D and 3-D matching scores, and Fig. 25 shows the receiver operating characteristic (ROC) curves. We can see that 3-D information is much more robust to illumination variation, scrabbling, and dirty than 2-D information.

\section{Database Establishment and Recognition Results}

A 3-D palmprint database has been established by using the developed 3-D palmprint imaging device. The database contains 6000 samples from 260 volunteers, including 182 males and 78 females. The 3-D palmprint samples were collected in two separated sessions, and in each session, about six samples were collected from each subject. The average time interval between the two sessions is two weeks. The original spatial resolution of the data is $768 \times 576$. After ROI extraction, the central part $(256 \times 256)$ is used for feature extraction and recognition. The $z$-value resolution of the data is 32 bits.

We performed two types of experiments on the established database: verification and identification. The experiments were performed by using the MCI, GCI, and ST features, as well as their score and feature-level fusion. In addition, to validate the 3-D palmprint recognition technique, we also compared it with the 2-D palmprint recognition method by using the 2-D palmprint images simultaneously collected in the 3-D palmprint acquisition process. The 2-D palmprint verification was performed

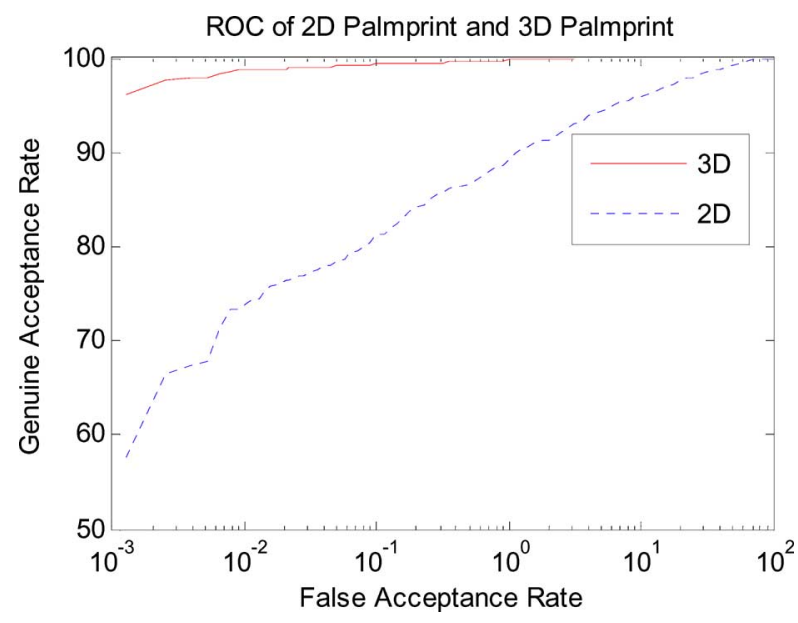

Fig. 25. ROC curves on the robustness test database.

by using the competitive coding method [5]. As we can see later in the experimental results, by fusing the 2-D and 3-D palmprint features, the highest recognition rate can be obtained.

Fig. 26 illustrates the genuine and imposter distributions of the verification results by 3 -D palmprint recognition. Fig. 26(a)(c) shows the curves by MCI, GCI, and ST features, respectively. Fig. 26(d) shows the curves by using their WM score-level fusion. The ROC curves by using the MCI, GCI, and ST features and their fusions (MAX, MIN, SUM, and MW) are shown in Fig. 27. The EER values, which are important indexes of the verification performance, are listed in Table IV, where the feature extraction and matching time by using different features are also listed. ${ }^{1}$ We see that the WM fusion achieves the smallest EER among all the schemes. From Fig. 27 and Table IV, we can see that all the fusion methods, except MIN, can achieve much better result than single feature matching. This is reasonable because more information usually leads to more accurate recognition.

Table IV also lists the EER of 2-D palmprint verification by using the competitive coding method [5]. We can see that 2-D palmprint recognition achieves much lower EER than 3-D

\footnotetext{
${ }^{1}$ The experiments were performed using Visual $\mathrm{C}++6.0$ on a $\mathrm{PC}$ with Windows XP Professional, Pentium 4 CPU of $2.66 \mathrm{GHz}$ and 1GB RAM.
} 


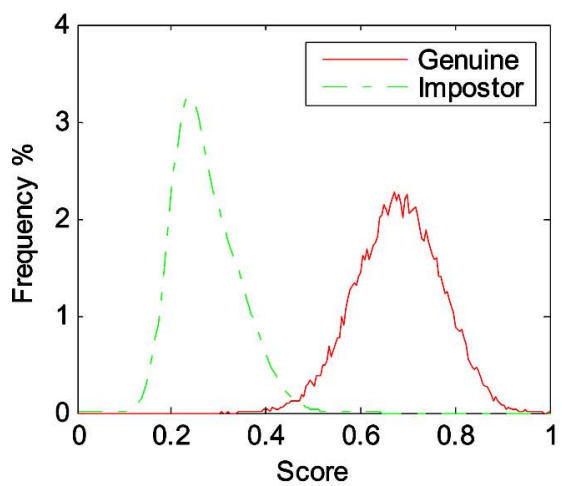

(a)

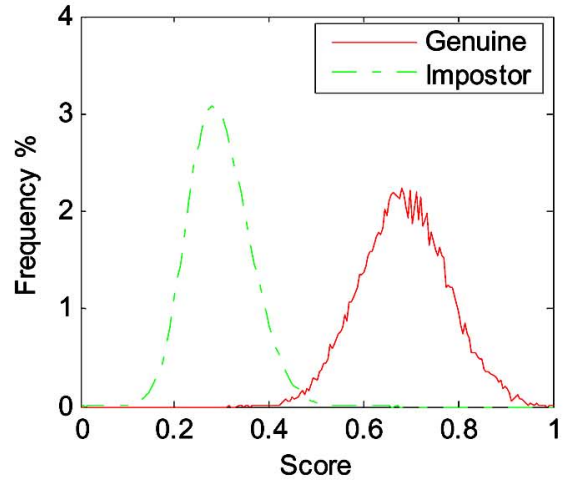

(c)

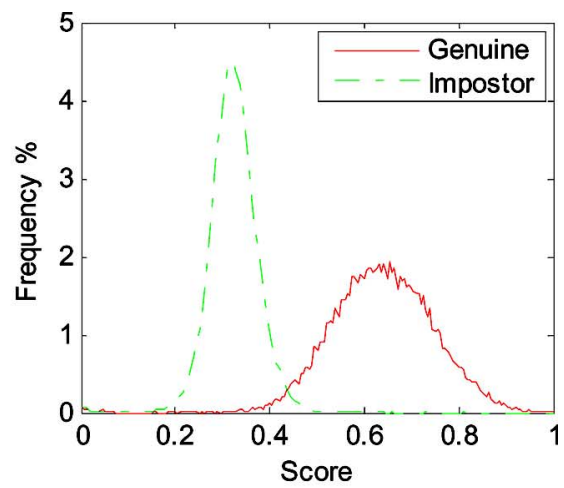

(b)

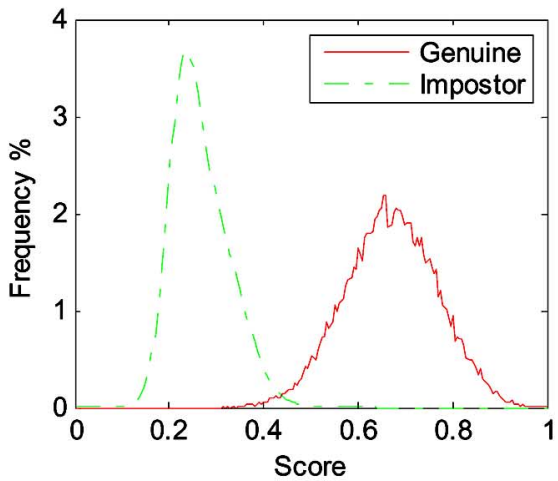

(d)

Fig. 26. Genuine and imposter distributions by MCI, GCI, ST features, and their WM fusion. (a) MCI. (b) GCI. (c) ST. (d) WM fusion.

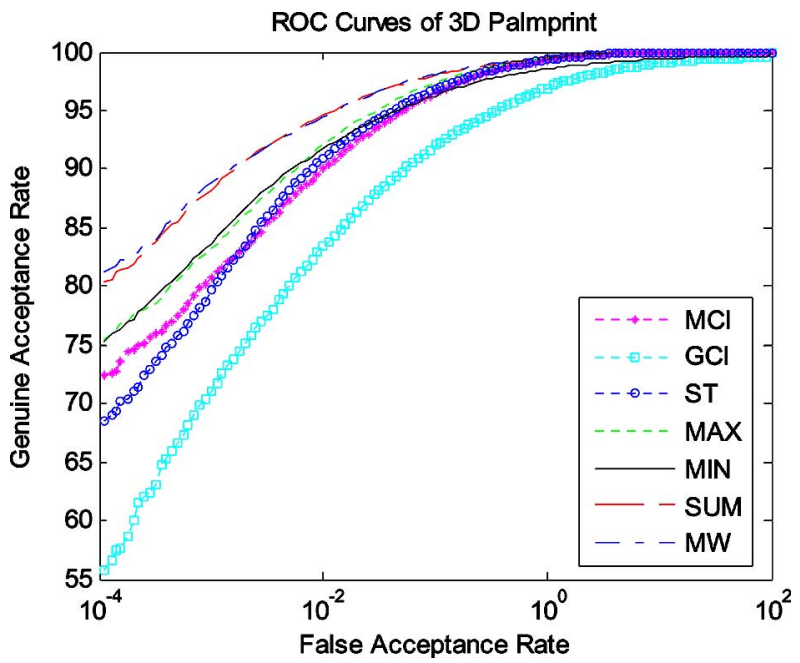

Fig. 27. ROC curves by different matching methods.

palmprint. This is mainly because the quality of 3-D palmprint data is not as good as that of 2-D palmprint. There is much corrupted noise in the data acquisition process and $z$-value accuracy needs further improvement. However, as shown in Sections V-A and $\mathrm{V}-\mathrm{B}$, the 3-D palmprint technique has higher antispoof capability and is more robust to illumination variations and scrabbling. There is much space to improve the 3-D palmprint data acquisition precision and the performance of feature extraction and matching algorithms. The 3-D palmprint recognition has great potential.
TABLE IV

EER VAlues, FeAture EXTRACTION Time, AND Matching Time By DIFFERENT METHODS

\begin{tabular}{|c|c|c|c|}
\hline & EER & Feature extraction time & Matching time \\
\hline MCI & $0.825 \%$ & $112 \mathrm{~ms}$ & $0.86 \mathrm{~ms}$ \\
\hline GCI & $2.059 \%$ & $112 \mathrm{~ms}$ & $0.86 \mathrm{~ms}$ \\
\hline ST & $0.791 \%$ & $115 \mathrm{~ms}$ & $3.76 \mathrm{~ms}$ \\
\hline MIN & $1.277 \%$ & $125 \mathrm{~ms}$ & $4.89 \mathrm{~ms}$ \\
\hline MAX & $0.714 \%$ & $125 \mathrm{~ms}$ & $4.89 \mathrm{~ms}$ \\
\hline SUM & $0.681 \%$ & $125 \mathrm{~ms}$ & $4.89 \mathrm{~ms}$ \\
\hline MW & $0.632 \%$ & $125 \mathrm{~ms}$ & $4.89 \mathrm{~ms}$ \\
\hline 2D & $0.126 \%$ & $97 \mathrm{~ms}$ & $0.15 \mathrm{~ms}$ \\
\hline 2D+3D SVM score level fusion & $0.068 \%$ & $189 \mathrm{~ms}$ & $5.01 \mathrm{~ms}$ \\
\hline 2D+3D feature level fusion & $0.059 \%$ & $245 \mathrm{~ms}$ & $0.15 \mathrm{~ms}$ \\
\hline
\end{tabular}

The 2-D and 3-D palmprints can be fused for higher accuracy. In Section IV-C, we have described the score-level fusion and feature-level fusion methods. After 2-D + 3-D feature-level fusion, we use the competitive coding method to extract the features from the enhanced 2-D ROI for matching. From Table IV, we see that the EERs valued by fusing 2-D and 3-D palmprint are much improved. The ROC curves by using 2-D features, $2-\mathrm{D}+3$-D score-level fusion, and 2-D + 3-D feature-level fusion are plotted in Fig. 28. It is clearly seen that 2-D and 3-D fusion achieves the best accuracy.

The experiments of identification were also conducted on the 3-D palmprint database. In identification, we do not know the class of the input palmprint but want to identify which class it belongs to. In the experiments, we let the first sample of each class in the database be the template and use the other samples as probes. Therefore, there are 5480 probes and 520 templates. 


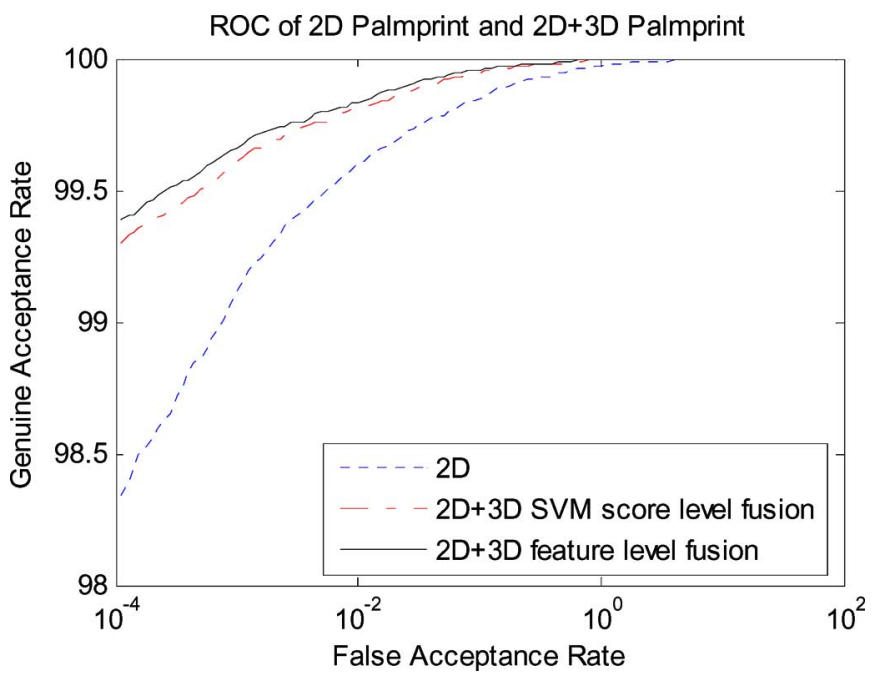

Fig. 28. ROC curves by 2-D palmprint and 2-D + 3-D palmprint.

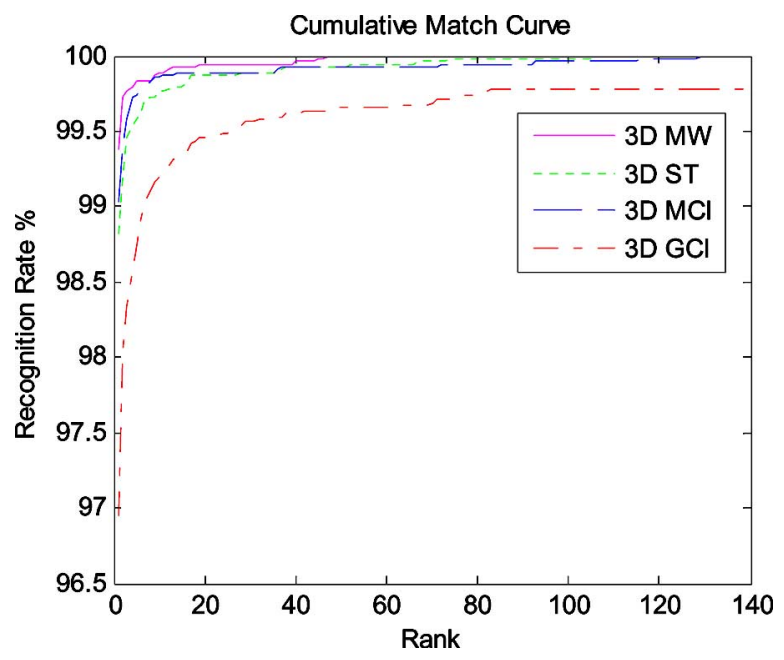

Fig. 29. CMC curves by different matching methods in identification.

TABLE V

Cumulative Matching Performance by Different Types of Features

\begin{tabular}{|c|c|c|c|c|}
\hline & MCI & GCI & ST & MW \\
\hline Rank-one recognition rate & $99.03 \%$ & $96.95 \%$ & $98.81 \%$ & $99.38 \%$ \\
\hline Lowest rank for perfect recognition & 129 & 473 & 106 & 47 \\
\hline
\end{tabular}

The probes were matched with all the template models, and for each probe, the matching results were ordered according to the matching scores. Then, we can get the cumulative match curves, as shown in Fig. 29. The cumulative matching performance, rank-one recognition rate, and lowest rank of perfect recognition (i.e., the lowest rank when the recognition rate reaches 100\%), are listed in Table V. From Fig. 29, we see that the performance of MW fusion is much better than the other schemes.

\section{CONCLUSION}

In this paper, we explored a new technique for palmprintbased biometrics: 3-D palmprint recognition. A structured-lightimaging-based 3-D palmprint data acquisition system was developed. After the 3-D palmprint image is captured, the ROI is extracted to roughly align the palm and remove the unnecessary cloud points. We then proposed the curvature-based feature extraction algorithms to extract the MCI, GCI, and ST features. A fast feature matching method and score-level and feature-level fusion strategies were used to classify the palmprints. A 3-D palmprint database with 6000 samples from 260 individuals was established, on which extensive verification and identification experiments were performed. The experimental results show that the 3-D palmprint technique can not only achieve high recognition rate, but also have high anticounterfeit capability and high robustness to illumination variations and serious scrabbling in the palm surface. In the future, more advanced and powerful feature extraction and matching techniques are to be developed for a better recognition performance.

\section{REFERENCES}

[1] R. M. Bolle, J. H. Connell, S. Pankanti, N. K. Ratha, and A. W. Senior, Guide to Biometrics. New York: Springer-Verlag, 2003.

[2] D. Zhang, Palmprint Authentication. Norwell, MA: Kluwer, 2004.

[3] D. Zhang and W. Shu, "Two novel characteristics in palmprint verification: Datum point invariance and line feature matching," Pattern Recognit., vol. 32, pp. 691-702, 1999.

[4] D. Zhang, A. W. K. Kong, J. You, and M. Wong, "On-line palmprint identification," IEEE Trans. Pattern Anal. Mach. Intell., vol. 25, no. 9, pp. 1041-1050, Sep. 2003.

[5] A. W. K. Kong and D. Zhang, "Competitive coding scheme for palmprint verification," in Proc. Int. Conf. Pattern Recognit., 2004, vol. 1, pp. 520523.

[6] L. Zhang and D. Zhang, "Characterization of palmprints by wavelet signatures via directional context modeling," IEEE Trans. Syst., Man, Cybern. B, Cybern., vol. 34, no. 3, pp. 1335-1347, Jun. 2004.

[7] A. W. K. Kong, D. Zhang, and M. Kamel, "Analysis of brute-force breakins of a palmprint authentication system," IEEE Trans. Syst., Man, Cybern. B, Cybern., vol. 36, no. 5, pp. 1201-1205, Oct. 2006.

[8] Z. N. Sun, T. N. Tan, Y. H. Wang, and S. Z. Li, "Ordinal palmprint representation for personal identification," in Proc. IEEE Int. Conf. Comput. Vis. Pattern Recognit., 2005, pp. 279-284.

[9] I. A. Kakadiaris, G. Passalis, G. Toderici, M. N. Murtuza, Y. L. Lu, N. Karampatziakis, and T. Theoharis, "Three-dimensional face recognition in the presence of facial expressions: An annotated deformable model approach," IEEE Trans. Pattern Anal. Mach. Intell., vol. 29, no. 4, pp. 640-649, Apr. 2007.

[10] C. Samir, A. Srivastava, and M. Daoudi, "Three-dimensional face recognition using shapes of facial curves," IEEE Trans. Pattern Anal. Mach. Intell., vol. 28, no. 11, pp. 1858-1863, Nov. 2006.

[11] B. Gokberk, H. Dutagaci, A. Ulas, L. Akarun, and B. Sankur, "Representation plurality and fusion for 3-D face recognition," IEEE Trans. Syst., Man, Cybern. B, Cybern., vol. 38, no. 1, pp. 155-173, Feb. 2008.

[12] H. Chen and B. Bhanu, "Human ear recognition in 3D," IEEE Trans. Pattern Anal. Mach. Intell., vol. 29, no. 4, pp. 718-737, Apr. 2007.

[13] P. Yan and K. W. Bowyer, "Biometric recognition using 3D ear shape," IEEE Trans. Pattern Anal. Mach. Intell., vol. 29, no. 8, pp. 1297-1308, Aug. 2007.

[14] V. Srinivassan and H. C. Liu, "Automated phase measuring profilometry of 3D diffuse object," Appl. Opt., vol. 23, no. 18, pp. 3105-3108, 1984.

[15] H. O. Saldner and J. M. Huntley, "Temporal phase unwrapping: Application to surface profiling of discontinuous objects," Appl. Opt., vol. 36, no. 13 , pp. 2770-2775, 1997.

[16] P. J. Besl and N. D. McKay, "A method for registration of 3-D shapes," IEEE Trans. Pattern Anal. Mach. Intell., vol. 14, no. 2, pp. 239-256, Feb. 1992.

[17] C. Hernandez, G. Vogiatzis, and R. Cipolla, "Multiview photometric stereo," IEEE Trans. Pattern Anal. Mach. Intell., vol. 30, no. 3, pp. 548554, Mar. 2008.

[18] R. Hartley, Multiple View Geometry in Computer Vision. Cambridge, U.K./New York: Cambridge Univ. Press, 2000.

[19] F. Blais, M. Rious, and J. A. Beraldin, "Practical considerations for a design of a high precision 3-D laser scanner system," Proc. SPIE, vol. 959, pp. 225-246, 1988. 
[20] G. C. Stockman, S. W. Chen, G. Hu, and N. Shrikhande, "Sensing and recognition of rigid objects using structured light," IEEE Control Syst. Mag., vol. 8, no. 3, pp. 14-22, Jun. 1988.

[21] S. M. Dunn, R. L. Keizer, and J. Yu, "Measuring the area and volume of the human body with structured light," IEEE Trans. Syst., Man, Cybern., vol. 19, no. 6, pp. 1350-1364, Nov./Dec. 1989.

[22] G. Hu and G. Stockman, "3-D surface solution using structured light and constraint propagation," IEEE Trans. Pattern Anal. Mach. Intell., vol. 11, no. 4, pp. 390-402, Apr. 1989.

[23] A. C. Sanderson, L. E. Weiss, and S. K. Nayar, "Structured highlight inspection of specular surfaces," IEEE Trans. Pattern Anal. Mach. Intell., vol. 10, no. 1, pp. 44-55, Jan. 1988.

[24] G. C. Stockman, S. W. Chen, G. Hu, and N. Shrikhande, "Sensing and recognition of rigid objects using structured light," IEEE Control Syst. Mag., vol. 8, no. 3, pp. 14-22, Jun. 1988.

[25] G. Sansoni, L. Biancardi, U. Minoni, and F. Docchio, "A novel, adaptive system for 3-D optical profilometry using a liquid crystal light projector," IEEE Trans. Instrum. Meas., vol. 43, no. 4, pp. 558-566, Aug. 1994.

[26] W. Kühnel, Differential Geometry: Curves-Surfaces-Manifolds. Providence, RI: American Mathematical Society, 2006.

[27] P. J. Besl and R. C. Jain, "Segmentation through variable-order surface fitting," IEEE Trans. Pattern Anal. Mach. Intell., vol. 10, no. 2, pp. 167192, Mar. 1988.

[28] D. L. Woodard and P. J. Flynn, "Finger surface as a biometric identifier," J. Comput. Vision Image Understanding (CVIU), vol. 100, no. 3, pp. 357384, Dec. 2005.

[29] R. Snelick, U. Uludag, A. Mink, M. Indovina, and A. Jain, "Large-scale evaluation of multimodal biometric authentication using state-of-the-art systems," IEEE Trans. Pattern Anal. Mach. Intell., vol. 27, no. 3, pp. 450455, Mar. 2005

[30] M. Indovina, U. Uludag, R. Snelick, A. Mink, and A. Jain, "Multimodal biometric authentication methods: A COTS approach," Proc. Workshop Multimodal User Authentication (MMUA 2003), pp. 99-106.

[31] G. Doddington, W. Liggett, A. Martin, M. Przybocki, and D. Reynolds, "Sheeps, goats, lambs and wolves: A statistical analysis of speaker performance in NIST 1998 speaker recognition evaluation," in Proc. ICSLD 1998, Nov., vol. 13, pp. 1-5.

[32] D. S. Huang, W. Jia, and D. Zhang, "Palmprint verification based on principal lines," Pattern Recognit., vol. 41, no. 4, pp. 1316-1328, Apr. 2008

[33] V. Vapnik, The Nature of Statistical Learning Theory. New York: Springer-Verlag, 1995.

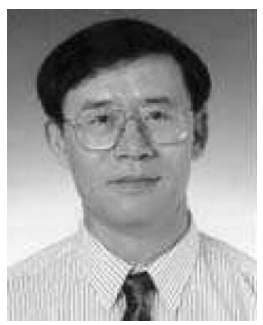

David Zhang (M'89-SM'95-F'08) received the Graduate degree in computer science from Peking University, Beijing, China, the M.Sc. and Ph.D. degrees in computer science from the Harbin Institute of Technology (HIT), Harbin, China, in 1982 and 1985, respectively, and the Ph.D. degree in electrical and computer engineering from the University of Waterloo, Waterloo, ON, Canada.

From 1986 to 1988, he was a Postdoctoral Fellow at Tsinghua University. He then became an Associate Professor at the Academia Sinica, Beijing. He is currently a Chair Professor at the Hong Kong Polytechnic University, Kowloon, Hong Kong, where he is also the Founding Director of the Biometrics Technology Centre (UGC/CRC) supported by the Hong Kong SAR Government in 1998. $\mathrm{He}$ is also a Visiting Chair Professor at Tsinghua University, and an Adjunct Professor at Shanghai Jiao Tong University, Harbin Institute of Technology, and the University of Waterloo. He is the Founder and the Editor-in-Chief of the International Journal of Image and Graphics (IJIG), and a Book Editor of the Springer International Series on Biometrics (KISB). He is the author or coauthor of more than ten books and 200 journal papers. He is an Associate Editor of more than ten international journals, including Pattern Recognition. His current research interests include biometrics, pattern recognition, image processing, medical diagnosis, etc.

Prof. Zhang is a Croucher Senior Research Fellow, a Distinguished Speaker of the IEEE Computer Society, and a Fellow of the International Association of Pattern Recognition (IAPR). He is also an Organizer of the International Conference on Biometrics Authentication (ICBA) and the Technical Committee Chair of the IEEE Computational Intelligence Society. He is an Associate Editor of the IEEE TRANSACTIONS ON SYSTEMS, MAN, AND CYBERNETICS-PART A: SYSTEMS AND HUMANS, and the IEEE TRANSACTIONS ON SYSTEMS, MAN, AND CYBERNETICS-PART C: APPLICATIONS AND REVIEWS.

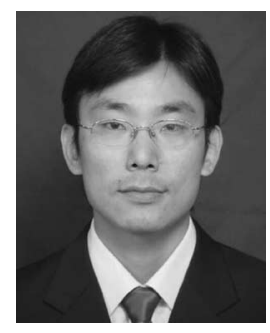

Guangming Lu received the Graduate degree in electrical engineering, the Master's degree in control theory and control engineering, and the Ph.D. degree in computer science and engineering from the Harbin Institute of Technology (HIT), Harbin, China, in 1998,2000 , and 2005 , respectively.

From 2005 to 2007, he was a Postdoctoral Fellow at Tsinghua University. He is currently an Associate Professor at the Biocomputing Research Center, Shenzhen Graduate School, HIT. His current research interests include pattern recognition, image processing, and automated biometric technologies and applications.

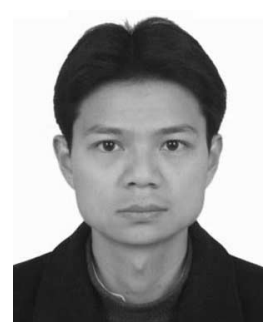

Wei Li (S'08) received the B.S. degree from Luoyang Institute of Technology, Luoyang, China, in 2001, and the M.S. degree in mechanical and electrical engineering from Henan University of Science and Technology, Luoyang, in 2005. He is currently working toward the Ph.D. degree at the Department of Automation, Shanghai Jiao Tong University, Shanghai, China.

His current research interests include pattern recognition, image processing, and graphics.

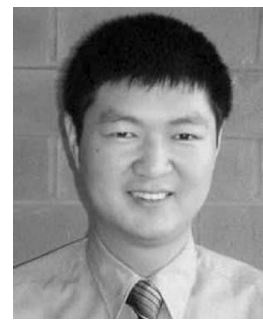

Lei Zhang (M'04) received the B.S. degree from Shenyang Institute of Aeronautical Engineering, Shenyang, China, in 1995, and the M.S. and Ph.D. degrees in electrical and engineering from Northwestern Polytechnical University, Xi'an, China, in 1998 and 2001, respectively.

From 2001 to 2002, he was a Research Associate in the Department of Computing, The Hong Kong Polytechnic University, Kowloon, Hong Kong, where he has been an Assistant Professor since January 2006 From January 2003 to January 2006, he was a Postdoctoral Fellow in the Department of Electrical and Computer Engineering, McMaster University, Hamilton, ON, Canada. His current research interests include image and video processing, biometrics, pattern recognition, multisensor data fusion, and optimal estimation theory, etc.

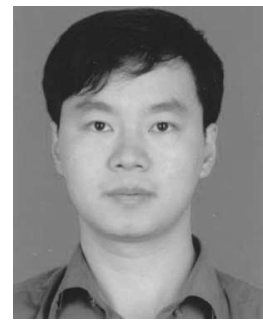

Nan Luo received the B.Eng. degree in mechanical design, manufacturing, and its automation from the Heilongjiang Science and Technology Institute, Harbin, China, in 2003.

Since 2004, he has been a Research Assistant at the Institute of Automation, Heilongiiang Academy of Sciences, Harbin. He is currently a Research Assistant at the Biometrics Research Centre, the Hong Kong Polytechnic University, Kowloon, Hong Kong. His current research interests include biometrics and computer vision. 